\title{
APPROCHES ET MÉTHODOLOGIES D'INSPIRATION VYGOTSKIENNES AUPRÈS DE L'HAUTE ÉCOLE SPECIALISÉE DE LA SUISSE ITALIENNE
}

\author{
Fulvio Poletti \\ fulvio.poletti@supsi.ch \\ Professeur/Professor \\ Responsable du Service pour la didactique universitaire et la formation des enseignants \\ Head of Higher Education and Teacher Training \\ (SEDIFO - http://www.supsi.ch/go/sedifo) \\ Haute école spécialisée de la Suisse italienne (SUPSI - www.supsi.ch) \\ University of Applied Sciences and Arts of Southern Switzerland
}

\section{Résumé}

Ce travail a pour but d'illustrer l'actualité de la pensée de Vygotsky par rapport à l'application de deux dispositifs pédagogiques - le Problem Based Learning (PBL) et le Problem Solving Coopératif (PSC) - dans l'une des huit universités professionnelles suisses, à savoir la Haute école spécialisée de la Suisse italienne (www.supsi.ch). La particularité de ces deux approches est de placer l'étudiant au centre du processus d'apprentissage, de sorte qu'il devienne le protagoniste dans l'acquisition des connaissances et des compétences nécessaires à l'exercice de la profession future ; nous nous sommes penchés sur les domaines social et sanitaire. Ces deux pratiques didactiques font l'objet d'une comparaison fructueuse avec l'élaboration théorique de Vygotsky, notamment avec certains concepts de son cadre conceptuel et méthodologique: l'importance du contexte historico-culturel pour la croissance du sujet, la Zone proximale de développement, la force heuristique de l'interaction sociale (dans un collectif/groupe) pour construire de nouveaux outils cognitifs par l'apprenant, la centralité de la métacognition pour l'apprentissage. La thèse fondamentale est que, bien que Vygotsky ait effectué ses recherches et observations surtout dans le monde de l'enfance, sa contribution peut très bien s'étendre à la condition de l'adulte qui apprend.

Mots clés : Problem Based Learning (PBL), Problem Solving Coopératif (PSC), contexte historico-culturel, apprentissage coopératif en groupe, Zone proximale de développement (ZPD), métacognition.

\begin{abstract}
This work aims at illustrating the topicality of Vygotsky's thinking with regard to the application of two educational devices - Problem Based Learning (PBL) and Problem Solving Cooperative (PSC) - at one of the eight Swiss professional universities: the University of Applied Sciences and
\end{abstract}


Arts of Southern Switzerland (www.supsi.ch). The distinctive feature of these approaches is that of placing the student at the heart of the learning process, so that $\mathrm{s} /$ he becomes the protagonist in the acquisition of the knowledge and skills necessary for the exercise of their future profession; we focus on social and health areas. These two didactic practices engender a fruitful comparison with Vygotsky's theoretical elaboration, especially with certain key concepts of his conceptual and methodological framework: the relevance of the historical-cultural context for the subject's growth, the zone of proximal development, the heuristic force of social interaction (in a community/group) allowing the learner to build new cognitive tools, the centrality of metacognition for learning. The main claim is that, although Vygotsky carried out his research and observations especially with regard to childhood, his contribution may very well extend to the condition of adult learning.

Keywords : Problem Based Learning (PBL), Problem Solving Cooperative (PSC), Culturalhistorical context, collaborative group learning, zone of proximal development (ZPD), metacognition.

\section{Note d'auteur}

Fulvio Poletti : https://orcid.org/0000-0003-2750-6645

Toute correspondance concernant cet article doit être adressée à (Fulvio Poletti), Servizio didattica e formazione dei docenti (SEDIFO), Direzione generale SUPSI, Le Gerre - Via Pobietta 11, CH-6928 Manno 


\section{Introduction}

Je travaille depuis longtemps auprès de la Haute école spécialisée (HES) de la Suisse italienne (SUPSI : $\underline{\text { www.supsi.ch })^{1}}$ et pendant plusieurs années, je me suis occupé de la formation des opérateurs du domaine social (éducateurs et assistantes sociaux) et du sanitaire (infirmiers, physiothérapeutes, ergothérapeutes). Dans ces contextes on a cherché à nous sensibiliser, entre autres, aux théories et aux indications méthodologiques de Vygostky et plus en général de l'Activisme pédagogique lié à plusieurs auteurs tels que Montessori, Decroly, Dewey, Freinet, Freire, etc. (Poletti, 2016).

Cet article vise à discuter de la validité et de la pertinence de la théorie historico-culturelle de Vygotsky pour et dans la formation au niveau académique, notamment en ce qui concerne les Hautes écoles spécialisés (HES). On aimerait montrer ici - en adoptant une posture phénoménologique et narrative et en récupérant des expériences de pratique formative - comment une telle conception est particulièrement adaptée pour les universités de ce genre, qui se caractérise, d'un côté, par la forte intégration dans le territoire de référence et, d'autre côté, par l'orientation professionnalisant de leur mission.

Tenu compte de cette propension à préparer adéquatement à l'exercice réel d'une profession, il s'est avéré fort important se référer aux expériences les plus proches des pratiques que les opérateurs en service effectuent couramment.

Pour cela nous avons fait appel à deux approches didactiques axés sur l'activation des sujets qui apprennent et qui deviennent ainsi protagonistes du processus d'acquisition des compétences visées. Il s'agit d'un côté du PBL - Problem Based Learning (Barrett, 2017; Barrows \& Tamblyn, 1980, 1980; Lotti, 2018; Poikela \& Poikela, 2005) en ce qui concerne les opérateurs sanitaires, respectivement du PSC - Problem Solving Coopératif (Baudrit, 2007; Galand et al., 2008; Johnson et al., 1994; Lalancette, 2014; Leone \& Prezza, 2016; Martini \& Sequi, 1999; Miller \& Rollnick, 2013; Steiner et al., 1999) touchant la formation des opérateurs sociaux.

Dans les deux cas, le principe est de confronter les étudiants avec des situations-problème concrètes qu'ils peuvent trouver dans leurs réalités professionnelles et pour lesquelles ils sont mis dans une posture de 'communauté de pratique' ou 'communauté d'apprentissage' qui prône la collaboration, la participation et la responsabilité partagée à l'intérieur des collectifs constitués (Carvalho-Filho et al., 2020; Fabbri, 2010; Lipari \& Valentini, 2013; Wenger, 1998, 2005). En vertu de cette intense et significative interaction (en se confrontant avec des taches et des problématiques authentiques) les équipes sont amenés à développer analyses, hypothèses et argumentations et par là construire un habitus attitudinal et cognitif axé sur la prise de conscience et la réflexion par rapport aux contextes et aux expériences professionnelles prises en considération.

Bien que les travaux de Vygotsky aient été élaborées par des recherches et observations effectuées surtout sur le monde des enfants, on parie ici sur le fait que ses idées et ses intuitions

\footnotetext{
${ }^{1}$ La Haute école spécialisée de la Suisse italienne est une des huit universités professionnelles suisses. Dotée d’un statut universitaire, elle est orientée vers la formation professionnelle et la recherche appliquée et participe au processus de Bologne. Ce qui signifie qu'elle délivre des titres d'études reconnus aussi bien en Suisse qu'au niveau européen. En fréquentant les cours Bachelor et Master de la SUPSI, les étudiants ont la possibilité d'enrichir leurs études académiques d'une précieuse pratique professionnelle, aspect fondamental pour l'entrée dans le monde du travail. L'enseignement, de haute qualité, est complété par des stages et des ateliers qui rapprochent les étudiants à la réalité de leur territoire et leur assure une position plus compétitive sur le plan international (Poletti, 2020).
} 
théoriques sont transférables même dans le panorama de la formation d'adultes, notamment dans les milieux universitaires, comme ceux des HES.

La thèse de fond de cet article est donc la suivante :

La contribution des théories vygotskiennes se révèle particulièrement actuelle et profitable

a) pour inspirer, soutenir et justifier épistémologiquement et méthodologiquement les propositions pédagogiques et didactiques que nous avons adoptées à la SUPSI, notamment le PBL et le PSC ;

b) pour les mettre en discussion et les dépasser dans le sens d'éviter de rester ancré à un activisme ou à un situationnisme naïf trop axé à la seule dimension pragmatique et expérientielle.

Avec ces suggestions et dans les intentions des formateurs, il ne s'agit donc pas simplement de se confronter à des expériences et des situations réelles, mais surtout de pousser la réflexion sur celles-ci, afin d'affiner la capacité de raisonnement et de parvenir à une pensée capable de généralisation et d'élaboration d'un cadre conceptuel pour fournir des points de repère pour organiser dûment un bagage professionnel.

Par la suite nous évoquons quelques-uns des apports particulièrement significatifs du cadre théorico-conceptuel du psychopédagogue russe, sans pour autant prétende à épuiser le spectre de des théories dont on pourrait s'inspirer, en les mettant en relation avec les deux paradigmes didactiques susmentionnés : le PBL et le PSC.

\section{Apports du cadre conceptuel de l'élaboration vygotskienne}

\section{Le.s sujet.s actif.s dans un contexte historico-culturel de référence}

Vygotsky souligne, dès l'âge le plus précoce, le dynamisme et la prédisposition proactive de toute personne en évolution et en formation. Au-delà de n'importe quel déterminisme, il attribue au sujet un rôle d'acteur et de protagoniste dans le processus de construction d'un bagage de connaissances, de compétences et d'habilitées pour affronter un monde existentiel et professionnel.

La création d'une toile de fond capable de donner du sens à l'apprentissage, avec l'intégration de certains contenus et aspects du contexte historico-culturel de référence en s'inspirant à Vygotsky, constitue une importante suggestion d'ordre méthodologique pour soumettre aux étudiants des situations-problème révélatrices et des tâches authentiques à résoudre, en stricte relation avec le futur professionnel qui les attend.

C'est justement le cas du PBL et du PSC que nous avons adopté dans la formation des opérateurs sociaux et sanitaires. Pour le pool de formateurs qui organisent les différentes séances de PBL et de PSC, il est utile de se référer à la vision historico-culturelle de Vygotsky, qui exhorte les formateurs à trouver des problématiques d'actualité qui soient paradigmatiques sur le plan professionnel, institutionnel et du cadre socioculturel de référence (selon une conception et une perspective systémique ${ }^{2}$ ). Il faut également calibrer leur degré de complexité, afin de s'adapter aux capacités de les affronter et de les dépasser de la part du corps étudiant (la finalité c'est de viser la Zone B de la ZPD).

\footnotetext{
${ }^{2}$ « Quand je parle de complexité, je me réfère au sens latin du mot complexus, ce qui est tissé ensemble. Les constituants sont différents, mais il faut voir comme dans une tapisserie la figure d'ensemble. Le vrai problème (de réforme de pensée) c'est que nous avons trop bien appris à séparer. Il faut mieux réapprendre à relier » (Morin, 1995, pp. 110-111; cf. Morin, 2008).
} 
Revue internationale du CRIRES: innover dans la tradition de Vygotsky (2021) ISSN 2291-6717, vol 5, no 2, 36-62

Approches et méthodologies d'inspiration Vygotskiennes auprès de l'Haute École Spécialisée de la Suisse Italienne

\section{La ZPD comme régulateur de l'apprentissage individuel et collectif}

Bien que la 'zone proximale de développement' (ZPD) ait été élaborée par Vygotsky surtout en prenant en considération la réalité de l'enfant, on peut aisément appliquer ce concept aussi à la situation des adultes engagés dans un processus de formation (Venet et al., 2016). Dans l'œuvre de Champy et Étévé (2005) se trouve une définition efficace et synthétique de ZPD, selon laquelle Vygotsky parvient :

À distinguer le niveau de développement actuel d'un sujet, déterminé par les épreuves et les problèmes qu'il peut résoudre seul, sans l'aide d'autrui, et qui correspond à l'exercice autonome et intériorisé des compétences cognitives, et son niveau de développement potentiel, déterminé par les épreuves et les problèmes qu'il n'est pas encore capable de résoudre seul, mais qu'il peut résoudre dans des situations de collaboration et d'interaction sociale. L'écart entre ces deux niveaux de développement définit ce que Vygotsky nomme la zone proximale de développement (ou zone de développement prochain) (p. 1051).

Par cette voie, nous adoptons le principe selon lequel l'apprentissage précède le développement, dans le sens où toute intervention pédagogique doit mirer à faire bouger le sujet vers des horizons évolutifs ultérieurs et pas se contenter d'agir sur le connu et sur l'acquis : dans ce cas, «l'apprentissage serait parfaitement inutile » s'il ne pousse l'individu «à dépasser ses propres limites » (Vygotsky, 1934b, p. 360).

Concernant notre hypothèse, le point essentiel consiste à dire que le processus de développement ne coïncide pas avec celui de l'apprentissage, mais que le processus de développement suit le processus d'apprentissage qui crée la zone du développement le plus proche. (Vygotsky, 1934a, p. 245)

L'apprentissage ne devient un apprentissage véritable que s'il devance le développement. (...). On a de bonnes raisons de supposer que le rôle de l'enseignement dans le développement de l'enfant consiste dans le fait que l'apprentissage crée la zone du développement le plus proche. (Vygotsky, 1933, pp. 168-169)

Selon cette ligne de pensée, on peut utiliser la ZPD pour procéder à une régulation calibrée des propositions didactiques à soumettre aux étudiants (Figure 1). 


\section{Figure 1}

Représentation figurative de la 'zone proximale de développement' de Lev S. Vygotsky

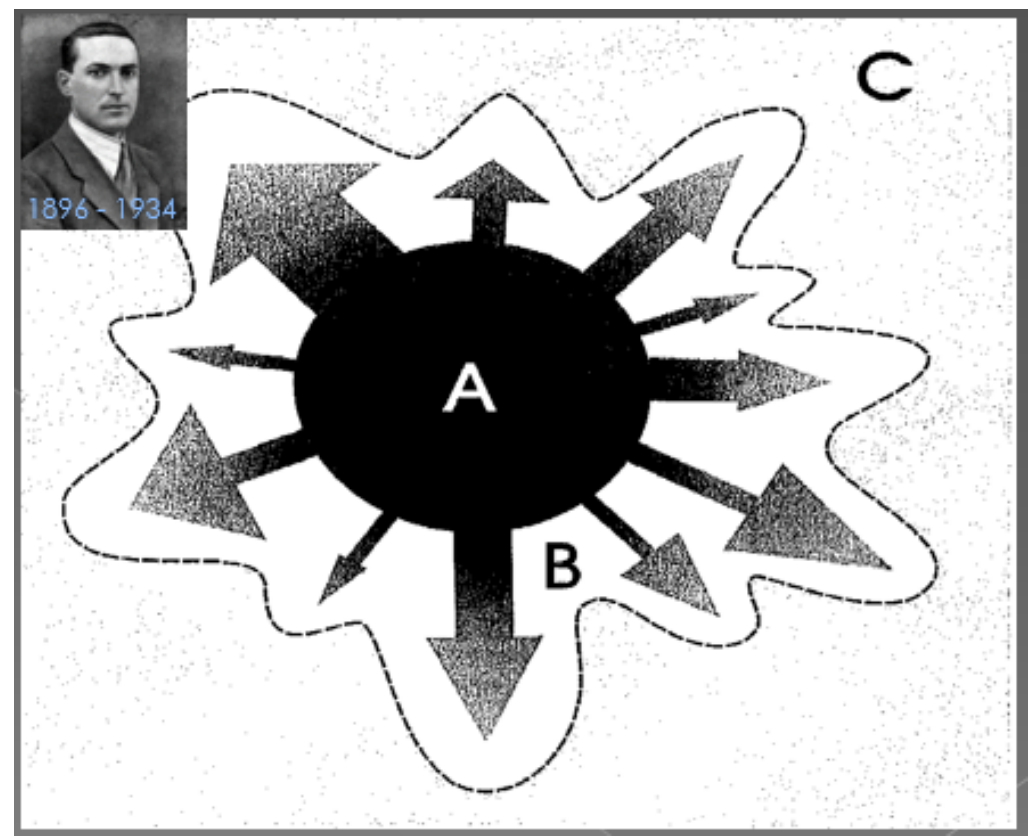

Zone A = l'apprenant peut effectuer la tâche sans aide, avec ses seules forces et ressources ;

Zone $\mathrm{B}=$ l'apprenant peut aborder la tâche avec l'aide/soutien d'un expert ou d'un pair plus habile (la différence des flèches représente les niveaux différents de prédispositions, maitrise et habilité du sujet par rapport aux activités/tâches humaines et aux défis/sollicitations du milieu);

Zone $\mathrm{C}=$ la tâche est au-dehors de la portée de l'apprenant.

Si on vise comme horizon évolutif de l'apprentissage la Zone A, on aura des 'objectifs inutiles', car le sujet en formation possède déjà les connaissances et les compétences pour y faire face. Si, par contre, on cherche à forcer en se situant dans la Zone C, on aura des 'objectifs impossibles' à rejoindre et à acquérir.

Se concentrer sur la surface $\mathrm{B}$ amène les enseignants à se pencher sur les différentes intelligences et sur les différentes propensions à apprendre ${ }^{3}$ qui entrent en jeu soit dans le parcours PBL et PSC de résolution du problème, soit plus en général par rapport à tout objet d'étude au centre du processus d'apprentissage.

Il s'avère donc crucial calibrer les choix didactiques par rapport à la ZPD, en organisant d'une façon efficace la difficulté représentée par les objectifs obstacles (Astolfi et al., 2008; Meirieu, 1990, 2017) à affronter et les aides au niveau des contenus et des indications méthodologiques, ainsi de déployer au maximum la réflexion métacognitive qui y est reliée.

L'apprentissage socialisé à l'intérieur de la ZPD comporte le développement de la conscience métacognitive (la réflexivité personnelle et de groupe) ainsi que les compétences transversales (les 'soft skills'), notamment dans les habilités sociocognitives : expression claire et

${ }^{3}$ Voire 'intelligences multiples' (Gardner, 2008) ; 'styles d'apprentissage' (Gaussel, 2016) ; 'différentiation pédagogique' (Forget, 2018; Service de soutien à la formation, 2015; Tobin \& Behling, 2018; Tomlinson, 2014) ; 'personnalisation de l'apprentissage' (Connac, 2017). 
bien argumentée de sa propre pensée, l'assomption d'un esprit critique solide et bien structuré, le partage de ressources et d'espaces communs, la gestion créative et positive des conflits, le respect des tours de parole dans les discussions.

Le tout parfaitement dans la mouvance des 'learning outcomes' et des finalités préfigurées par les 'descripteurs de Dublin': délimitant l'horizon des résultats d'apprentissage souhaités à l'issue d'un cycle d'études universitaires.

\section{Figure 2}

Les descripteurs génériques du cadre de qualification de l'EES

Les descripteurs génériques du cadre de qualification de l’EEES (« descripteurs de Dublin »)

\begin{tabular}{|c|c|c|c|}
\hline & Bachelier & Master & Doctorat \\
\hline Connaissances et compréhension & $\begin{array}{l}\text { Connaissances et } \\
\text { compréhension dans un } \\
\text { domaine d'études (basées sur } \\
\text { des ouvrages scientifiques et } \\
\text { des savoirs issus de la } \\
\text { recherche). }\end{array}$ & $\begin{array}{l}\text { Connaissances pour } \\
\text { développer et mettre en } \\
\text { œuvre des idées } \\
\text { originales (innovation). } \\
\text { Souvent par la recherche. }\end{array}$ & $\begin{array}{l}\text { Compréhension en } \\
\text { profondeur et maîtrise des } \\
\text { méthodes de recherche. }\end{array}$ \\
\hline Application des connaissances & $\begin{array}{l}\text { Utilisation de façon } \\
\text { professionnelle des } \\
\text { connaissances et compétences } \\
\text { acquises et aptitude à élaborer } \\
\text { et développer, dans un domaine } \\
\text { d'études, des arguments et des } \\
\text { solutions à des problématiques. }\end{array}$ & $\begin{array}{l}\text { Application - à des } \\
\text { situations nouvelles ou } \\
\text { pluridisciplinaires - des } \\
\text { connaissances et } \\
\text { compétences ainsi que } \\
\text { des capacités à résoudre } \\
\text { des problèmes. }\end{array}$ & $\begin{array}{l}\text { Contribution à la production, } \\
\text { l'analyse critique et } \\
\text { l'évaluation de } \\
\text { connaissances nouvelles et } \\
\text { complexes. }\end{array}$ \\
\hline Analyse critique & $\begin{array}{l}\text { Capacité à récolter et traiter des } \\
\text { données significatives pour } \\
\text { poser des jugements critiques } \\
\text { (problématiques sociales, } \\
\text { scientifiques ou éthiques) }\end{array}$ & $\begin{array}{l}\text { Capacité à poser des } \\
\text { jugements critiques en } \\
\text { gérant la complexité et } \\
\text { l'information manquante. }\end{array}$ & $\begin{array}{l}\text { Capacité à concevoir, } \\
\text { mettre en œuvre et réguler } \\
\text { un processus de recherche. }\end{array}$ \\
\hline Communication & $\begin{array}{l}\text { Capacité à communiquer à des } \\
\text { spécialistes comme à des } \\
\text { profanes des informations, des } \\
\text { idées, des problèmes et des } \\
\text { solutions. }\end{array}$ & $\begin{array}{l}\text { Capacité à communiquer } \\
\text { à des spécialistes comme } \\
\text { à des profanes les } \\
\text { fondements des } \\
\text { connaissances. }\end{array}$ & $\begin{array}{l}\text { Capacité à communiquer } \\
\text { dans une communauté de } \\
\text { chercheurs ou d'experts. }\end{array}$ \\
\hline Autodidaxie & $\begin{array}{l}\text { Capacité d'apprentissage pour } \\
\text { poursuivre la formation } \\
\text { universitaire, avec un fort degré } \\
\text { d'autonomie. }\end{array}$ & $\begin{array}{l}\text { Capacité d'apprentissage } \\
\text { de manière largement } \\
\text { autonome (self-directed). }\end{array}$ & $\begin{array}{l}\text { Promotion de la } \\
\text { connaissance dans la } \\
\text { société. }\end{array}$ \\
\hline
\end{tabular}

présentation ppt de Ph.Parmentier (2008) : learning outcomes, de la contrainte au projet.

Source : http://sbottani.net/lab/wp-content/uploads/2018/11/Cadre-de-qualification-Descripteursde-Dublin.pdf

\section{La force et la valeur heuristique de l'apprentissage coopératif en groupe}

Les suggestions théoriques et méthodologiques de Vygotsky amènent à considérer d'une façon particulière le climat et l'ambiance générales dans lesquels le parcours de formation se déroule. Elles s'inscrivent parfaitement dans la ligne historique de l'Activisme pédagogique, prônant pour que les étudiants puissent vivre des expériences positives, immersives et engageantes, en les projetant dans une dimension formative active et collaborative.

Pour cela, le climat dans lequel se produit le processus d'enseignement et d'apprentissage se révèle de grande importance et il se concrétise avec des attitudes, des comportements et des relations de qualité et de respect, où le rôle du professeur est celui de point de repère, de ressource 
à disposition, de disponibilité à l'écoute et à l'aide indirecte [cf. «éducation négative» selon la conception de J.J. Rousseau $\left.(1762 / 1992)^{4}\right]$ : soutien discret, sans se substituer à l'apprenant dans son parcours de croissance intérieure, intellectuelle, sociale et culturelle. Il va donc assumer une fonction de metteur en scène et de catalyseur prônant à l'action directe, créatrice et propulsive des sujets en formation, auxquels on garantit une large marge de manœuvre et de liberté pour s'épanouir et accroitre leurs connaissances. Nous sommes ici parfaitement en ligne avec la pensée vygotskienne : « Le seul éducateur capable de créer de nouvelles réactions dans l'organisme est l'expérience. (...) L'éducation doit être organisée de telle sorte que l'élève ne soit pas éduqué, mais que l'élève s'éduque par lui-même » (Vygotskij, 2006, p. 93 et 94).

Dans ce cadre, il est facile de reconnaitre les prémisses et les conditions visant à la promotion d'une éducation à la citoyenneté active et participative, dans un climat caractérisé par une approche démocratique et coopérative.

Avec cette toile de fond on peut concevoir surtout le PSC : terrain fertile pour pratiquer la confrontation dans le respect réciproque, l'argumentation pondérée, la négociation dans l'effort commun de trouver des interprétations partagées et des propositions de solution aux problématiques soumises au collectif des étudiants, divisés par groupes d'étude. Sans pour autant méconnaître la possibilité de tensions et de conflits, qui font également partie des interactions humaines et professionnelles normales et qui sont donc susceptibles de constituer un matériel supplémentaire pour apprendre à accroître ses compétences.

Le contexte envisagé est orienté à fournir et à garantir la marge d'autonomie la plus large possible aux apprenants, ainsi qu'ils puissent expérimenter concrètement la force ou la puissance heuristique de l'apprentissage social, conçu comme prioritaire pour le développement cognitif dans et par le groupe.

C'est justement la longue et intense fréquentation à travers la simulation d'une véritable équipe de travailleurs sociaux, pendant tout un semestre, qui fonctionne comme terrains formatifs particulièrement significatif et stimulant pour perfectionner ses prérogatives professionnelles.

De nombreuses recherches contemporaines, rejoignant celles de Vygotsky, ont montré en quoi l'interaction sociale peut conduire l'apprenant à bâtir de nouveaux instruments cognitifs lui permettant, à leur tour, de participer à des interactions plus élaborées, favorisant de nouveaux progrès intellectuels. Ces études précisent les effets des interactions entre pairs. Ces recherches font apparaître que, sous certaines conditions, non seulement des groupes d'apprenants confrontés à un problème utilisent des stratégies supérieures à celles de chaque individu mais aussi que ces progrès peuvent être intériorisés, de manière stable, par chacun. Cette appropriation advient si les points de vue des partenaires, en étant ni trop éloignés ni trop lacunaires, supportent une confrontation bénéfique sur le plan cognitif (Grangeat, 2016, p. 136).

\footnotetext{
4 « Rousseau propose une manière originale d'éduquer : l'éducation négative. Il s'agit d'une éducation par la nature, une éducation qui refuse les opinions et la morale ; une éducation qui n'est pas basée sur les connaissances déclaratives, car l'apprentissage doit venir de l'expérience des choses et non de la connaissance par les mots. L'éducation négative laisse donc la nature agir. (...) si l'éducateur laisse la nature agir, il n'est pas pour autant réduit à un rôle totalement passif. En réalité, tout en suivant scrupuleusement la nature, c'est tout de même lui qui choisit à la fois le contenu (expériences et observations) et le moment propice pour le mettre à la disposition de l'enfant [du sujet en formation]. En fin de compte, la pédagogie de Rousseau peut être qualifiée de négative dans la mesure où elle propose d'intervenir le moins possible auprès de l'enfant afin de le laisser réaliser ses propres expériences » (Martineau \& Buysse, 2016).
} 
Revue internationale du CRIRES: innover dans la tradition de Vygotsky (2021) ISSN 2291-6717, vol 5, no 2, 36-62 Approches et méthodologies d'inspiration Vygotskiennes auprès de l'Haute École Spécialisée de la Suisse Italienne

\section{Le développement de la métacognition dans le cadre d'une communauté de pratique ou d'apprentissage}

Une des grandes contributions de Vygotsky est la croyance ferme à la force propulsive et expansive de l'enseignement, capable de stimuler la croissance intellectuelle du sujet en formation : «À la différence de l'ancienne théorie, la théorie de la zone du développement le plus proche se traduit par une formule qui est l'exact opposé et qui dit que le seul bon enseignement est celui qui précède le développement » (Vygotsky, 1934a, p. 240).

Est ici affirmé d'une manière très puissante ce que Philippe Meirieu $(1990$; 2017) appelle le 'principe d'éducabilité' (fil rouge de la posture pédagogique du mouvement de l'École nouvelle ou de l'Activisme pédagogique du siècle passé) par lequel on parie sur la possibilité et la capacité d'épanouissement de chaque et toute personne, peu importe son extraction sociale et familiale, ses limites ou déficits psychophysiques, sa provenance ethnico-culturelle.

Bien que Vygotsky distingue clairement les formes spécifiques d'apprentissage de l'enfant de celles de l'adulte, ainsi que leur rapport - tout à fait particulier - avec le développement, on ne peut pas conclure par là que pour lui, en tant qu'adulte, ne se vérifie plus aucune transformation significative de la pensée. Cette hypostatisation ne serait pas compatible avec le dynamisme et le caractère de dépassement continu - à travers le support de l'observation, l'expérimentation et la recherche continue - témoignés pour toute sa (courte) vie par Vygotsky.

$\mathrm{Si}$, à l'âge adulte, la configuration biopsychique atteinte est achevée et il n'y a plus de révolution et de changements fondamentaux sur le plan structurel des fonctions psychiques supérieures, du point de vue qualitatif, nous pouvons penser qu'il y a des marges de manœuvre pour considérer comment un certain apprentissage puisse provoquer un enrichissement et voire une réorganisation de la manière de penser le monde par un sujet qui se trouve dans cette phase de l'existence.

Il n'aura en effet pas échappé au lecteur que l'influence de Vygotsky a largement dépassé les limites de la psychologie de l'enfant et qu'elle a inspiré un certain nombre de textes et d'écrits portant sur le développement et la formation de l'adulte (Yvon, 2011, p. 379).

Si on se penche sur la formation professionnalisant qui constitue la mission des HES, on pourra y étendre et appliquer - avec quand même les nécessaires ajustements - les considérations vygotskiennes : «l'apprentissage n'est pas équivalent au développement (...) Chaque apprentissage est une source de développement qui réveille les processus qui, sans lui, ne peuvent pas apparaître » (Vygotsky, 1934a, p. 243).

Dans une communauté d'apprentissage ou de pratique $e^{5}$ orientée professionnellement (simulation d'un travail en équipe) il s'agit pour les membres de mettre en mots et en énoncés adéquats les conceptualisations formulées dans l'action. Par des situations-problème on cherche à dégager un processus heuristique visant à l'élaboration de concepts à la fois pragmatiques ('incarnés' ou 'en-acte' dans des contextes réels, en analogie ou en résonance avec les compétences

\footnotetext{
${ }^{5}$ En s'inspirant à l'hypothèse de fond de Wenger (très proche de la pensée de Vygotsky), par laquelle l'engagement dans une pratique sociale est le processus fondamental par lequel on apprend et on évolue en tant qu'être humain, on a cherché de la décliner, avec les ajustements opportuns, dans le cadre de la formation universitaire HES. II s'agit pourtant, dans notre cas, d'un dispositif formatif axé sur une conception d'apprentissage expérientiel caractérisé par la négociation et co-construction de concepts, compétences, méthodologies dans la confrontation de situationsproblèmes concrètes/réelles issues du contexte historico-culturel d'appartenance. "Le concept de pratique est associé à 'faire', mais pas strictement en tant que tel. C'est 'faire' dans un contexte historique et social qui donne une structure et une signification à ce qu'on accomplit. En ce sens, une pratique est toujours sociale. »; " la pratique doit être interprétée comme un processus d'apprentissage » (Wenger, 2005, p. 53 et 55).
} 
qu'on cherche à cultiver), et scientifiques (avec l'invitation à récupérer tout le bagage théorique l'encyclopédie formative - afférent aux cours suivi dans son propre curriculum).

Avec la confrontation directe à des problématiques/tâches issues de réalités professionnelles et avec l'amplification de l'interaction socioconstructiviste dans un collectif de travail, les étudiants sont poussés à mettre en circulation leurs représentations psycho-sociales, leurs besoins, leurs difficultés, leurs conflits, leurs acquisitions culturelles, etc. De plus, ils sont exhortés à mobiliser toutes les ressources du milieu circonstant, en s'appropriant la connaissance de l'organisation et du fonctionnement des instances sociales et institutionnelles importantes du contexte historico-culturel de référence. On poursuit ainsi une appropriation toujours plus consciente des 'systèmes sémiotiques' : artefacts socialement construits, élaborés et transmis d'une génération à l'autre, aussi à travers la profession.

En faisant interagir la dimension psycho-sociale, historico-culturelle et cognitive, l'approche fondée sur la communauté de pratique ou d'apprentissage vise à construire une pensée bien structurée et un habitus professionnel capable de discernement et de créer des connexions créatives et inventives pour résoudre des problèmes complexes qui caractérisent les contextes professionnels actuels. Tout ça correspond à des compétences clés pour les professionnels de la santé et les travailleurs sociaux, c'est-à-dire des domaines axés sur la relation et la communication, où les capacités de médiation, négociation et résolution de problèmes sont fondamentales.

\section{Deux approches dans le cadre de la formation académique professionnalisant}

\section{Le Problem Based Learning (PBL)}

Les principales composantes et dimensions de l'approche par problèmes sont présentées ci-après de manière figurative et synthétique.

\section{Figure 3}

Dimensions et composantes $d u P B L$

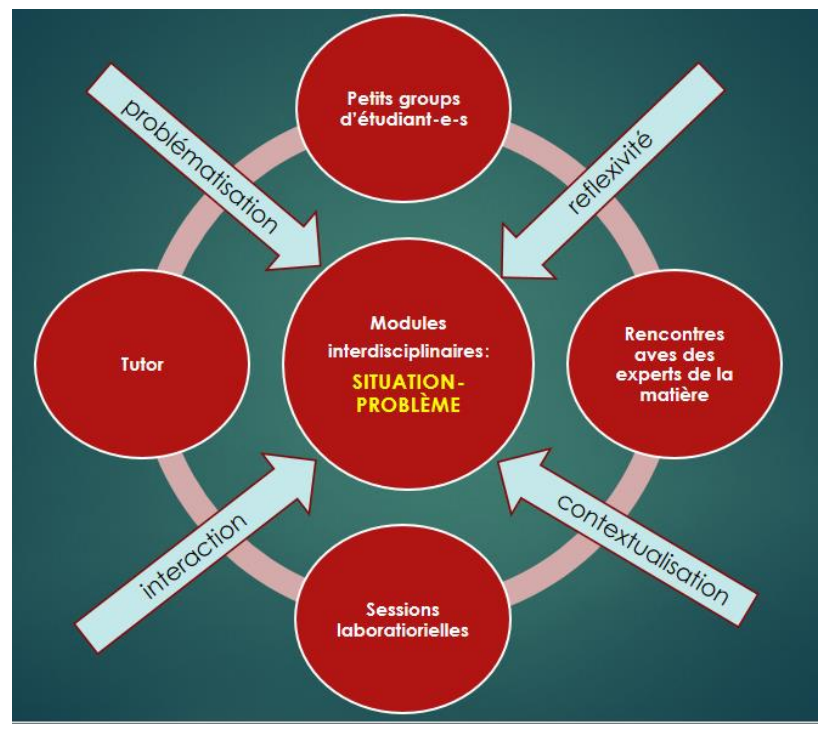


L’apprentissage est ancré à un processus de caractère constructiviste plutôt que réceptifreproductif où au centre on dispose une situation problématique à traiter dans une perspective interdisciplinaire. Le dispositif, appliqué dans un cadre séminaire-laboratoire (groupe d'étude idéalement constitués de 8 à 12 membres) vise à promouvoir une façon d'apprendre dégagée et orientée par un problème significatif et réel, issu du monde du travail, capable d'engendrer la motivation - en mobilisant besoins et intérêts des sujets en formation - et d'enraciner les connaissances et les compétences en profondeur.

Les facteurs contextuels et sociaux influent significativement sur l'apprentissage. Il s'agit donc de prôner sur : des situations-problème réelles et des tâches authentiques (Sambell et al., 2013), capables d'encourager l'enrichissement d'une 'pensée située' (Mottier Lopez, 2016) à faire jouer dans un 'setting' laboratoire convenant ; le rôle socioconstructiviste du groupe des apprenants, en stimulant des confrontations et des conflits sociocognitifs avec aussi l'activation ou la consultation d'experts sur l'objet d'étude pris en considération; la récupération des connaissances et d'expériences préalables (préconceptions) à faire évoluer vers conceptions toujours plus raffinées et robustes scientifiquement.

La métacognition est un grand levier de l'apprentissage du fait qu'elle induit une amélioration cognitive marquante. On envisage par cette voie l'assomption d'une posture heuristique marquée par les opérations suivantes : se poser constamment des questions, se préfigurer des objectifs de formation, choisir des stratégies résolutives adéquates, évaluer l'efficacité de son parcours de construction des connaissances et perfectionnement des habilités et compétences personnelles.

Pour toutes ces raisons, nous avons adopté l'approche PBL pour dépasser un enseignement purement traditionnel et notionnel en faveur d'un autre, plus dynamique, mettant au centre le processus d'apprentissage stimulé par des situations authentiques ancrées à la pratique professionnelle. La méthode prévoit l'analyse du problème assigné à travers huit étapes de travail disposées séquentiellement : voir le schéma 3 ci-dessous. 
Revue internationale du CRIRES: innover dans la tradition de Vygotsky (2021) ISSN 2291-6717, vol 5, no 2, 36-62 Approches et méthodologies d'inspiration Vygotskiennes auprès de l'Haute École Spécialisée de la Suisse Italienne

\section{Figure 4}

\section{Confrontation entre enseignement traditionnel et PBL}

\section{ENSEIGNEMENT TRADITIONNEL}

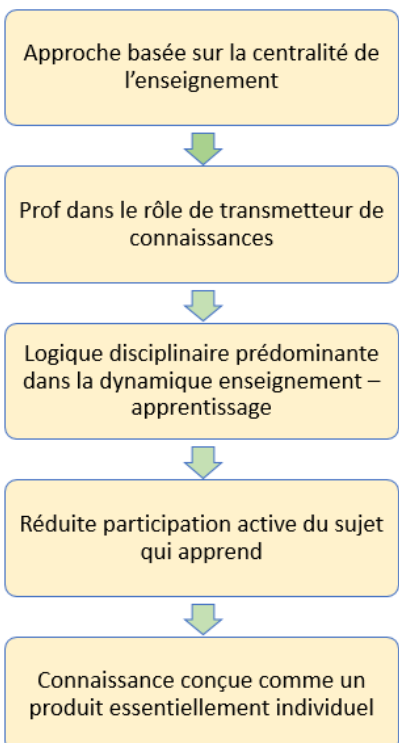

PROBLEM BASED LEARNING

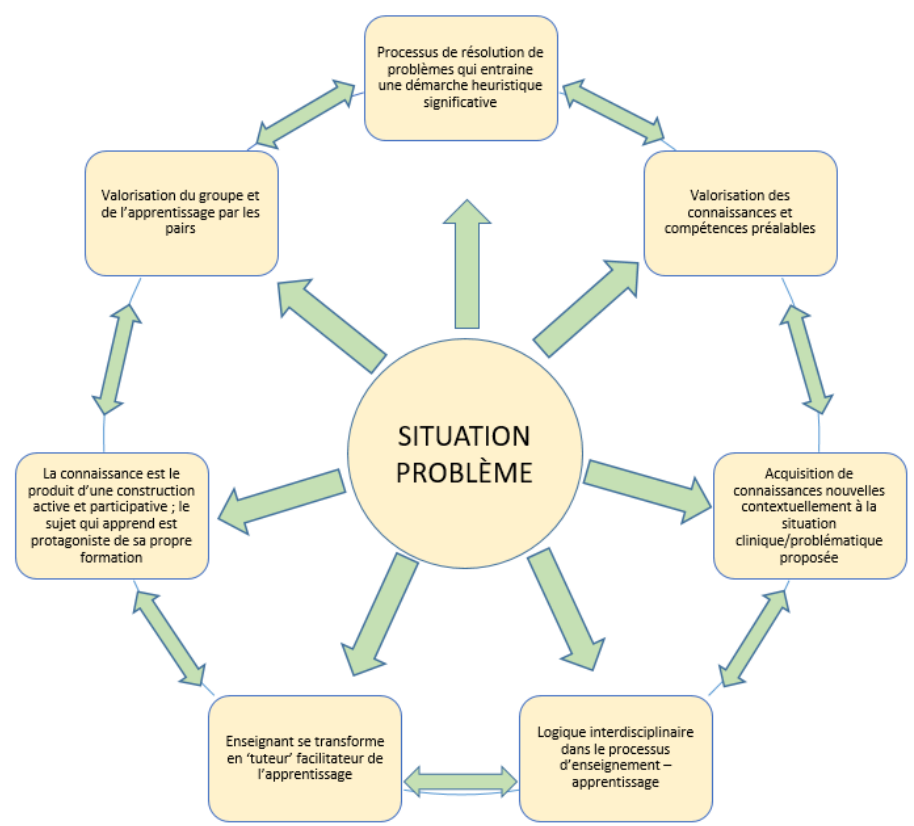

\section{Figure 5}

\section{Étapes séquentielles du Problem Based Learning 6}

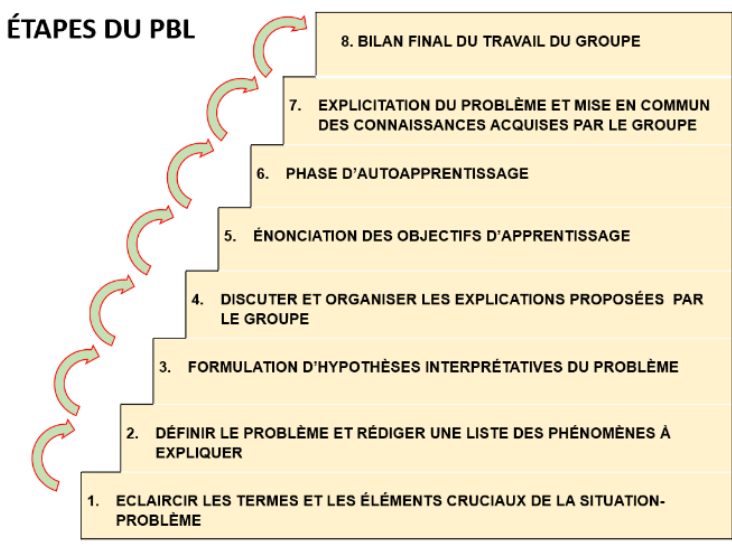

${ }^{6}$ Pour la description des étapes voir :

https://www.unige.ch/medecine/fr/enseignement1/bachelor-et-master-en-medecine-humaine/formatsapprentissage/app/appen8videos/ 
On peut présenter les questions à aborder ou les situations-problème en utilisant une pluralité de langages et vecteurs communicatifs : textes écrits, dessins, schémas, cartes conceptuelles, storytelling, vidéos, présentations avec support numérique, témoignages, etc.

Le problème à soumettre au corps étudiant pour dégager le processus de découverte devrait être : captivant, intrigant, stimulant ; d'actualité, réaliste et authentique ; pertinent par rapport aux objectifs globaux du cours ou du module ; formulé d'une manière claire et synthétique ; à la portée des capacités cognitives des étudiants ; susceptible de générer un éventail d'hypothèses, de pistes heuristiques, de découvertes, de sujets à développer ; proposé de manière à prendre un temps d'étude adéquat.

Le rôle de l'enseignant se traduit en 'tuteur': il devrait soutenir le processus d'apprentissage en assurant « un style maïeutique, en tirant de l'étudiant initialement des réponses imprécises et fortement caractérisées sur le plan émotionnel, pour l'aider progressivement à se confronter à la structure rationnelle du problème » (Binetti \& Alloni, 2004, p. 97).

Par contre, les rôles principaux prévus pour les participants aux groupes d'étude sont : animateur, qui gère les étapes des travaux, favorise la recherche du consensus, stimule la participation ; secrétaire, qui note au tableau les éléments marquants qui ressortent de la discussion et de la confrontation d'idées ; verbaliste, qui transcrit (sur support papier ou informatique) ce qui est indiqué sur le tableau, en intégrant d'autres éléments contextuels et de référence, et le rend accessible au groupe.

Voici la division en quatre sections distinguées du tableau, suggéré par Howard Barrows, pour orienter et faciliter l'organisation du travail pendant les sessions de PBL (Barrows \& Tamblyn, 1980; Barrows \& Wee Keng Neo, 2007).

\section{Tableau 1}

Les sections thématiques pour organiser la démarche heuristique, selon Barrows

\begin{tabular}{|c|c|c|c|}
\hline $\begin{array}{l}\text { Hypothèses } \\
\text { interprétatives }\end{array}$ & $\begin{array}{l}\text { Informations, } \\
\text { donnés }\end{array}$ & Arguments d'étude & Plan d'action \\
\hline $\begin{array}{l}\text { Inventaire des causes } \\
\text { possibles du } \\
\text { problème et mise au } \\
\text { point de solutions } \\
\text { appropriées pour y } \\
\text { remédier }\end{array}$ & $\begin{array}{l}\text { Éléments } \\
\text { d'information jugés } \\
\text { importants sur le } \\
\text { problème, tirés de sa } \\
\text { présentation et mis en } \\
\text { relation avec les } \\
\text { hypothèses }\end{array}$ & $\begin{array}{l}\text { Liste des } \\
\text { connaissances à } \\
\text { acquérir et des } \\
\text { compétences à } \\
\text { mobiliser pour } \\
\text { comprendre et } \\
\text { résoudre le problème }\end{array}$ & $\begin{array}{l}\text { Liste des opérations } \\
\text { et procédures que le } \\
\text { groupe doit mettre en } \\
\text { œuvre pour atteindre } \\
\text { les objectifs }\end{array}$ \\
\hline
\end{tabular}

Durant le parcours de résolution du problème assigné, sont prévues des séances où les sousgroupes sont regroupés pour suivre des leçons d'encadrement et de développement conceptuel ou méthodologique tenues par des spécialistes en la matière.

\section{Le Problem Solving Coopératif (PSC)}

Même l'approche de Problem Solving est finalisée à la résolution de problèmes en accordant beaucoup d'attention au processus qui, dans notre cas, est encore plus important que le produit réalisé. 
Il est destiné aux futurs travailleurs sociaux qui se trouveront dans l'exercice de la profession à travailler souvent dans une configuration d'équipe de pairs interdisciplinaire. Il s'avère donc éducatif pour développer un apprentissage en groupe du type coopératif qui pousse à travailler ensemble selon une posture d'équipe.

On peut représenter le PSC comme une procédure séquentielle constituée par six phases, à concevoir d'une façon circulaire et systémique :

\section{Figure 6}

Les phases constitutives du Problem Solving Coopératif

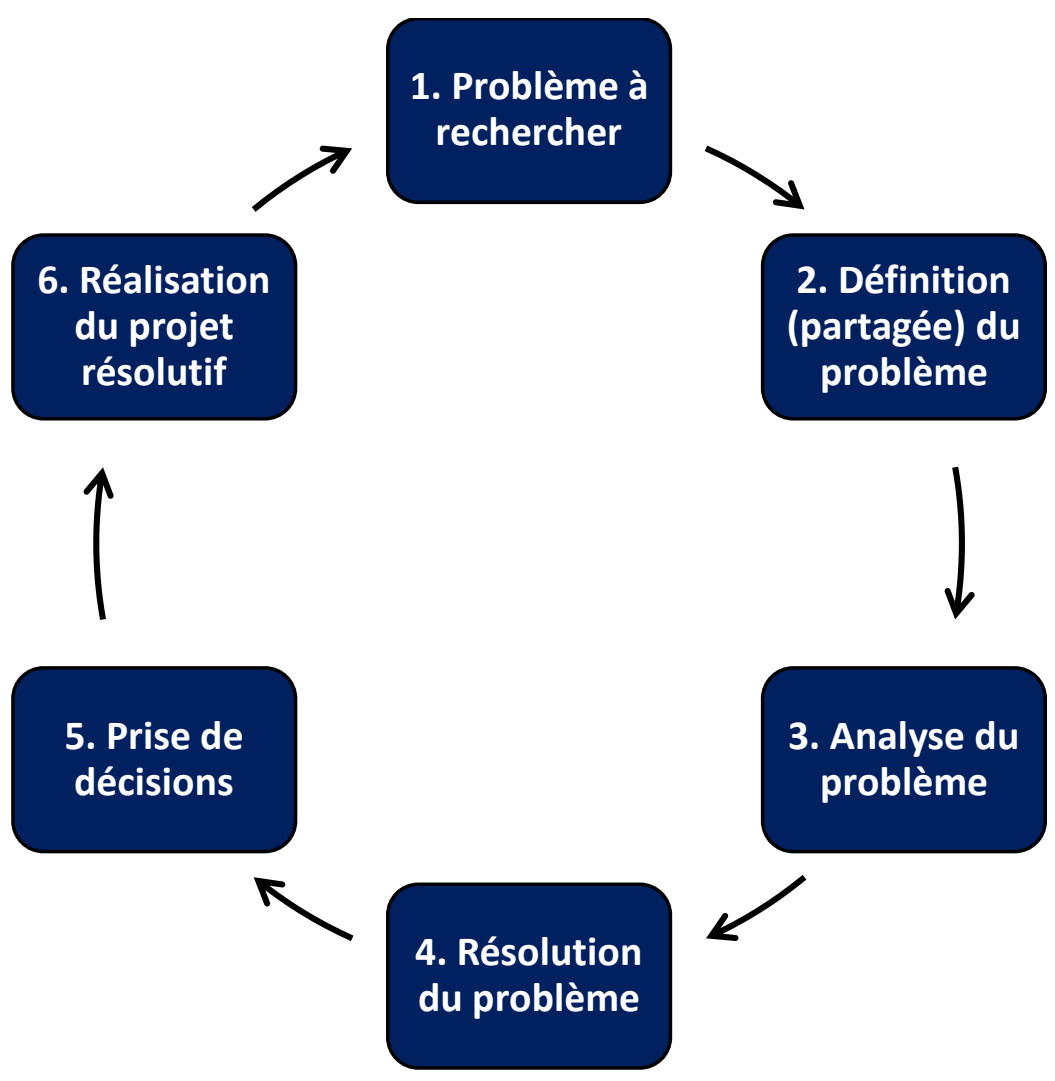

Problème à rechercher : connaissance de la situation contextuelle à aborder et naissance de l'idée de projet.

Dans ce sens, le passage d'une perception subjective du problème entre les composants du groupe impliqué dans la démarche est fondamental (ici aussi, idéalement, composé entre 8-12 individus), à la reconnaissance partagée du problème.

La question clé à se poser est : Y a-t-il un problème, pour 'nous'?

Définition du problème : Processus d'élaboration d'une définition partagée du problème à affronter entre les acteurs concernés et construction de l'idée de projet.

Il faut à ce point délimiter le périmètre et cerner les composants principaux du nœud problématique identifié, en reconnaissant les éléments constitutifs et en faisant surgir les questions émergentes. 
La question clé est : Lequel est-il le problème, qu’on a reconnu dans la phase précédente ? Dans ces deux phases initiales, cruciales dans l'économie de tout le parcours, il est essentiel donc de bien définir la problématique principale et d'éclaircir au maximum l'objet d'étude, évitant de se précipiter trop vite sur les mesures résolutives. La tentation est forte dans ce sens, mais c'est à l'habilité de l'animateur (enseignant) de recentrer le groupe sur la tâche et de bien saisir la problématique, par consensus dans le collectif de travail, avant de se dédier à trouver des remèdes et des solutions.

Analyse du problème : perfectionnement de l'idée du projet en partageant l'étude approfondie du problème.

La question clé est : Quels sont les éléments qui constituent le problème et ses implications?

Ce n'est qu'à partir de la quatrième phase du PSC que l'on entre dans l'hypothèse de réponses opérationnelles.

Résolution du problème : négociation en équipe d'un projet commun pour résoudre le problème considéré.

La question clé est : Que peut-on faire?

Prise de décisions : processus décisionnel et de négociations avec d'autres points du réseau et avec des partenaires du territoire de référence et, par conséquent, redéfinition/ajustement du projet envisagé.

La question clé est : Qu'est-ce qu'on fait?

Réalisation du projet résolutif : passage à l'action et vérification des résultats.

Le PSC comporte une activité cognitive qui implique à la fois des processus de pensée divergents et créatifs (comme dans les procédures de brainstorming) et des processus de pensée convergents (lorsqu'il est demandé d'évaluer de manière rationnelle les différentes hypothèses et de choisir la solution la plus adéquate et la plus réalisable par rapport à la situation problématique prise en considération).

Activer des modalités de participation et de partage dans la définition du problème et dans les autres phases du PSC aide les personnes impliquées à s'ouvrir à une multiplicité de démarches méthodologiques et à des stratégies de résolution. Dans cette démarche de dialogue et de confrontation continue, interviennent constamment des aspects rationnels et irrationnels ou inconscients comme la culture de référence, les histoires autobiographiques, les valeurs personnelles, les connaissances préalables, les expériences passées, les émotions et bien plus encore.

S'il n'est pas correctement géré, le parcours de résolution des problèmes réalisés en groupe peut devenir pénible et dispersif. Voici par la suite, dans le tableau 2, les variables que plus que d'autres peuvent jouer en négatif dans le processus et le rôle que l'enseignant/animateur peut assumer pour y faire face. 
Revue internationale du CRIRES: innover dans la tradition de Vygotsky (2021) ISSN 2291-6717, vol 5, no 2, 36-62 Approches et méthodologies d'inspiration Vygotskiennes auprès de l'Haute École Spécialisée de la Suisse Italienne

\section{Tableau 2}

Situations problématiques possibles et les moyens de les résoudre par les formateurs

\begin{tabular}{|l|l|}
\hline \multicolumn{1}{|c|}{ inconvénients / impasses } & \multicolumn{1}{c|}{ rôle de l'animateur/enseignant } \\
\hline la connaissance limitée du problème du PSC & $\begin{array}{l}\text { il s'agit d'introduire des informations et des } \\
\text { connaissances, de stimuler des recherches, de conseiller } \\
\text { des parcours, des stratégies et des matériaux }\end{array}$ \\
\hline le manque de compétences méthodologiques & $\begin{array}{l}\text { il convient dans ce cas de fournir des informations } \\
\text { complémentaires sur les techniques d'organisation du } \\
\text { groupe de travail, les connaissances sur les méthodes de } \\
\text { résolution des problèmes et de conception participative, } \\
\text { soit maintenir en vie un processus de soutien et de } \\
\text { réflexion et d'évaluation continues }\end{array}$ \\
\hline $\begin{array}{l}\text { une sur } \\
\text { l'expérimentation pendant le parcours de formation }\end{array}$ \\
$\begin{array}{l}\text { fermeture, des rivalités et des jalousies, une compétition } \\
\text { excessive, un manque de sentiment d'appartenance) qui } \\
\text { peut entraver la marche du groupe }\end{array}$ & $\begin{array}{l}\text { il est nécessaire de suivre l'évolution des dynamiques } \\
\text { relationnelles en intervenant sur le groupe (par la } \\
\text { personnes) et sur les individus (par des interventions } \\
\text { pendant les rencontres et des feedbacks écrits en les } \\
\text { intégrant avec les carnets e-learning personnels) }\end{array}$ \\
\hline
\end{tabular}

Le PBL est appliqué chez nous pour aborder des contenus interdisciplinaires assez circonscrits pendant quelques semaines (normalement deux ou trois), où on applique la méthodologie susmentionnée notamment dans la formation des futurs professionnels de la santé, c'est-à-dire dans le domaine des soins infirmières, de la physiothérapie et de l'ergothérapie [voir un exemple à l'Annexe A].

Le PSC se déroule par contre sur l'arc d'un semestre entier, dans la troisième année du 'bachelor' concernant la formation des opérateurs sociaux (éducateurs et assistants sociaux en interaction parmi eux), constituant un terrain formatif particulièrement significatif car il s'agit d'un contexte d'apprentissage où faire confluer, mobiliser et réélaborer idéalement tout le bagage conceptuel et méthodologique de la formation préalable (les cours de la première et de la deuxième année) en vue de construire des compétences spécifiques inhérentes à la profession choisie.

En effet, le module PSC vise à favoriser le développement de connaissances fondamentales pour la profession et de compétences d'action professionnelles.

Ses objectifs sont de : 1) renforcer les connaissances sur l'intervention sociale : concepts et méthodologies du processus de résolution des problèmes, en s'appuyant sur les modules suivis précédemment ; 2) observer le cas au moyen de concepts et de méthodologies et le/la décrire, l'analyser et l'expliquer de manière systématique ; 3 ) reconnaître les possibilités et les limites de l'intervention professionnelle ; 4) participer de manière active et constructive au travail d'équipe ; 5) reconnaître la nécessité de la collaboration interprofessionnelle ; 6) organiser et planifier de manière autonome son travail en tenant compte des besoins et des ressources internes à l'équipe.

Dans cette direction, travailler en groupe dans une perspective collaborative signifie acquérir des habilités organisationnelles et procédurales de grande envergure pour la construction d'une professionnalité solide et efficace dans le cadre des métiers socio-éducatifs. Elles se configurent de la façon suivante : organiser les rôles jugés nécessaires pour la réunion (au moins un coordinateur et un verbaliste, mais aussi des autres fonctions si retenues utiles au bon déroulement du travail commun), partager les attentes et la signification qui leur sont attribuées et les respecter au cours de la réunion ; structurer le temps de la réunion et contrôler la prévision 
établie ; décider des techniques de discussion et de décision à utiliser ; définir de manière partagée et explicite l'objectif spécifique de la réunion ; parvenir aux conclusions par le consensus le plus large et le plus participatif possible.

Certains processus relationnels, tels que ; l'équilibre entre les intérêts individuels et les motivations du groupe ; le degré de cohésion du team ; l'interdépendance effective des membres du groupe ; la qualité du climat et de la communication interne ; l'attitude à l'égard du débat et la compréhension des phénomènes non positifs et la capacité de résolution des conflits sont essentiels.

Sur le plan pratique, les participants ont été confrontés à deux cas différents en raison de leur complexité.

Le premier cas [voire un exemple à l'Annexe B] avait pour objectif de faire expérimenter aux étudiants l'ensemble du processus sans le souci d'arriver à un 'produit' définitif ou complet. Le but était de vérifier les doutes et les critiques que les groupes auraient pu rencontrer, en mettant en évidence leurs défaillances et les difficultés de méthode et de processus.

À ce stade, la tâche des enseignants (assignés un par groupe) a été de guider, étape par étape, le travail des étudiants sous trois aspects : le respect rigoureux du processus de résolution des problèmes (définition $\rightarrow$ analyse $\rightarrow$ recherche de solutions $\rightarrow$ décision), avec une attention particulière à la phase de définition et d'analyse du problème ; le soin apporté au choix des méthodologies de discussion et de décision par l'explicitation et le partage des procédures (rôles, techniques, temps); la réflexion sur les processus du groupe, avec une relecture commune de ce qui s'est passé entre les participants.

Avant le lancement de la deuxième phase, un simple modèle de 'procès-verbal' adopté par chaque groupe a été présenté en plénière, ainsi qu'un journal de bord individuel en ligne sur une base hebdomadaire.

Le second cas [voire un exemple à l'Annexe C] de complexité croissante par rapport au premier, a été soumis au groupe d'étudiants afin qu'ils l'abordent avec la plus grande autonomie. Ceci a favorisé une plus grande responsabilisation du collectif dans son ensemble et des individus qui le composaient. Le 'contrat pédagogique' avec les formateurs prévoyait que chaque groupe s'organisait de manière autonome pour réaliser une séance hebdomadaire au-dehors des cours, sans la présence de l'enseignant attribué, auquel il fallait tout de même faire parvenir le procèsverbal de la séance. Cette demande a été faite pour mettre les partenaires dans une position de proximité par rapport au fonctionnement d'une véritable équipe de professionnels (tâche authentique).

Le journal de bord s'est révélé un instrument particulièrement fécond pour engendrer la 'pratique réflexive' (Perrenoud, s.d.; Schön, 1994, 1996) : le fill rouge de toute notre formation, ayant pour objectif la tension vers la figure d'un opérateur qui maintient l'effort constant d'interroger et de s'interroger sur la signification des actions, des comportements, des modes de communication et des choix méthodologiques qui le concernent et du contexte dans lequel il opère.

Nous avons cherché à documenter, tant la dynamique relationnelle et procédurale au sein des groupes, que la capacité de réflexion que chaque participant mettait en œuvre individuellement par rapport à l'expérience effectuée.

La préoccupation des enseignants a été de suivre constamment, mais très discrètement pour pas se substituer aux apprenants - le déploiement des facultés et des potentialités du sujet et du collectif en formation, mettant à l'œuvre une observation 'indirecte' minutieuse en ce qui concerne les dynamiques relationnelles et méthodologiques qui se déroulent dans les séances (autogérées) des groupes au travail, accompagnées par une interaction biunivoque - par 
l'intermédiaire du journal de bord personnel - entre 'tuteur' et étudiant singulier, pour aider ce dernier à prendre conscience de ses potentialités et fragilités, afin de l'inciter à renforcer les premières et à compenser/dépasser les secondes.

La posture réflexive et la 'pensé critique' (Motoi, 2016) sont une constante qui traverse l'ensemble du processus de formation professionnelle académique, ultérieurement stimulée par les feedbacks des formateurs/tuteurs donnés à chaque étudiant, afin de renforcer la métacognition souhaitée. Pour cette voie on envisage de poursuivre une formalisation scientifique et professionnellement adéquate aux idées naïves ou acerbes et aux concepts pragmatiques, par l'acquisition ou la co-construction d'un lexique et d'un langage interprétatifs/compréhensifs bien équipés et appropriés pour aborder la complexité de la vie professionnelle. La systématisation des concepts pragmatiques peut «jouer dans ce sens un grand rôle dans le développement des compétences professionnelles» (Yvon, 2011, p. 396). En effet, l'apprentissage " crée non seulement des processus psychiques complètement nouveaux qui sont le résultat direct de l'assimilation du langage écrit, de la grammaire, des concepts scientifiques, mais réorganise également les formes de l'activité psychique établies antérieurement. D'où l'aphorisme de Vygotsky : «La conscience réflexive arrive par la porte des concepts scientifiques » (Elkonine, 2011, p. 315).

À la fin du semestre, chaque groupe a présenté en plénière les éléments marquants, tant au niveau du contenu que du processus communicatif-relationnel, de son parcours et de son projet.

Par la suite, les groupes ont dressé un bilan de la formation par rapport à l'apprentissage de la méthodologie de résolution de problèmes, à l'expérience de l'organisation du groupe, à l'autoévaluation des modalités de collaboration mises en œuvre par les membres.

Le rôle de l'enseignant, de guide ponctuel de chaque passage (phase 1), est passé à celui de 'tuteur' : observateur, consultant sur la demande et appui au besoin. En tant qu'observateur, il a vérifié les progrès du groupe sur le plan du contenu et du processus, en les liant aux réflexions des étudiants contenues dans les carnets hebdomadaires, rédigés individuellement et chargés sur la plate-forme électronique Moodle. En tant que consultant, il a répondu à des questions concernant la recherche du matériel, des informations spécifiques non connues des étudiants, des demandes d'aide explicites sur le plan du contenu ou de la méthode.

Les interventions des enseignants/tuteurs ont été principalement destinées à: permettre la focalisation ou la réorientation du problème ; faire prendre conscience au groupe de la phase du processus dans laquelle il se trouvait pour permettre son développement correct et ultérieur; rappeler ou préciser l'utilisation de méthodes de discussion spécifiques ; faciliter l'explicitation de certains troubles de communication qui risquaient d'entraîner des conflits relationnels; suggérer des pistes pour la présentation des résultats du parcours.

Pour la certification du module, il est prévu une implication responsabilisante du corps étudiant dans l'évaluation : répartie à cinquante pour cent entre formateurs et étudiants.

En ce qui concerne l'évaluation du module par les étudiants, un bon résultat au niveau d'appréciation globale peut être souligné.

Voici quelques extraits de verbatim :

L'autogestion, même dans les moments les plus difficiles, a été un bon exercice parce que chacun a pris ses responsabilités et on a construit le projet comme des professionnels.

Pouvoir mettre en pratique ce que nous avons appris les années précédentes. Aussi, j'ai apprécié que nous pouvions expérimenter le travail en équipe, exactement comme cela se produira une fois que vous entrez dans le monde du travail. 
Revue internationale du CRIRES: innover dans la tradition de Vygotsky (2021) ISSN 2291-6717, vol 5, no 2, 36-62

Approches et méthodologies d'inspiration Vygotskiennes auprès de l'Haute École Spécialisée de la Suisse Italienne

J'ai apprécié qu'une partie des réunions se déroulent de manière autonome, sans la présence de l'enseignant, car je crois que cela a permis au groupe d'agir de manière moins contrôlée et rigide, en faisant ressortir certains aspects intéressants du travail en groupe.

La mise en pratique de la théorie, et la mise en jeu de nos compétences d'équipe.

Les aspects critiques sont tout concentrés sur la charge de travail jugé de certains comme excessive :

Les réunions hors classe sont très exigeantes en matière de temps, surtout vu la charge des travaux requis globalement pendant le semestre impliqué.

Faire moins de rencontres. Se réunir pendant toutes ces heures conduit probablement à allonger chaque phase du travail et parfois même à ne plus se supporter.

\section{Conclusion}

Il ne s'agit pas de procéder à une célébration hagiographique de l'élaboration de Vygotsky, mais plutôt de s'en inspirer pour actualiser ses contributions par rapport à nos pratiques formatives.

En ce qui nous concerne, ses suggestions et ses indications les plus stimulantes amènent des contributions fort intéressantes dans plusieurs directions :

1) Elles contribuent à inspirer, soutenir et à justifier épistémologiquement et méthodologiquement les deux approches prises en considération, c'est-à-dire le PBL et le PSC, ainsi de rendre l'apprentissage académique professionnalisant plus dynamique, actif et proche de la réalité des opérateurs sur le terrain, de façon cohérente avec le paradigme des compétences (Chauvigné \& Coulet, 2010; Hébrard, 2013; Le Boterf, 2018; Poumay et al., 2017; Scott, 2015; Tardif, 2006).

2) Elles permettent de corriger ces approches professionnalisantes en récupérant la dimension conceptuelle et métacognitive pour éviter un aplatissement sur la seule pratique et un certain empirisme expérientiel. Vygotsky, bien qu'il prône un apprentissage axé sur l'expérience personnelle du sujet en formation, nous exhorte à déployer au maximum le développement et le perfectionnement des facultés cognitives supérieures, en se détachant de l'expérience pratique pour l'élaborer vers la construction de cadres conceptuels toujours plus sophistiqués et riches. C'est par cette voie que l'on cultive de plus en plus la métacognition : l'autorégulation de son propre apprentissage, la réflexion critique et une plus grande conscience sur son propre travail, la capacité de généraliser et d'abstraction, le transfert des connaissances et des compétences à d'autres contextes et situations que ceux dans lesquels l'expérience de formation a eu lieu ou s'est déroulée (Bråten, 1991; Chick, 2013; Fox \& Riconscente, 2008; Yale Center for Teaching and Learning, s.d.) .

3) Elles sollicitent, en outre, à adopter une 'posture de recherche' (du style rechercheaction-formation) (Charlier, 2006; Lavoie et al., 1996) visant à systématiser nos réalisations expérientielles et cognitives. Cette mouvance heuristique nous incite à remettre constamment en cause les acquis obtenus, en nous poussant vers des objectifs de plus en plus perfectionnés sur le plan des connaissances et des compétences. Ceci afin de lire avec une certaine amplitude de vue éthico-sociale et de profondeur épistémologique, la réalité socioculturelle dans laquelle nous agissons professionnellement et dans laquelle nous sommes insérés existentiellement. 
La prise de ces orientations et l'adoption de ces mesures, sur stimulation de la pensée vygotskienne, permettrait de rendre le PBL et le PSC scientifiquement plus solides et didactiquement plus efficaces dans les processus de formation destinés aux futurs professionnels.

Voici selon nous, une façon de tenir vivante la mémoire d'un géant de la psychologie, comme l'avait défini Jérôme Bruner. En effet, Vygotsky a encore beaucoup de choses à nous dire et à nous suggérer, et il est toujours pertinent d'interroger avec acuité et intelligence ses puissantes intuitions.

\section{Références}

Astolfi, J.-P., Darot, É., Ginsburg, Y., \& Toussaint, J. (2008). Obstacle, objectif-obstacle. Dans J.-P. Astolfi, É. Darot, Y. Ginsburg, \& J. Toussaint (Eds.), Mots-clés de la didactique des sciences (pp. 121129). De Boeck Supérieur.

Barrett, T. (2017). A New Model of Problem-based learning: Inspiring Concepts, Practice Strategies and Case Studies from Higher Education. All Ireland Society for Higher Education (AISHE).

Barrows, H. S., \& Tamblyn, R. M. (1980). Problem-based learning: An approach to medical education. Springer.

Barrows, H. S., \& Wee Keng Neo, L. (2007). Principles and Practice of aPBL. Pearson Education South Asia.

Baudrit, A. (2007). Apprentissage coopératif/Apprentissage collaboratif: D'un comparatisme conventionnel à un comparatisme critique: Les Sciences de l'éducation-Pour l'Ére nouvelle, Vol. 40(1), 115-136.

Binetti, P., \& Alloni, R. (2004). Modi e modelli del tutorato: La formazione come alleanza. Ed. Magi.

Bråten, I. (1991). Vygotsky as Precursor to Metacognitive Theory: I. The Concept of Metacognition and Its Roots. Scandinavian Journal of Educational Research, 35(3), 179-192.

Champy, P., \& Étévé, C. (2005). Dictionnaire encyclopédique de l'éducation et de la formation (3 éd.). Retz.

Carvalho-Filho, M. A. de, Tio, R. A., \& Steinert, Y. (2020). Twelve tips for implementing a community of practice for faculty development. Medical Teacher, 42(2), 143-149.

Charlier, B. (2006). Parcours de recherche-action-formation. Revue des sciences de l'éducation, 31(2), 259272.

Chauvigné, C., \& Coulet, J.-C. (2010). L'approche par compétences: Un nouveau paradigme pour la pédagogie universitaire ? Revue française de pédagogie. Recherches en éducation, 172, 15-28.

Chick, N. (2013). Metacognition. Vanderbilt University Center for Teaching. https://cft.vanderbilt.edu/guides-sub-pages/metacognition/

Connac, S. (2017). La personnalisation des apprentissages: Agir face à l'hétérogénéité, à l'école et au collège (3e éd). ESF éditeur.

Dewey, J. (1897). My Pedagogic Creed. 54, 77-80.

Dewey, J. (2011). Démocratie et éducation: Suivi de Expérience et Éducation (éd. or. 1916/1938). Armand Colin.

Elkonine, D. B. (2011). Le problème de l'enseignement et du développement dans les travaux de L. S. Vzgotskij. Dans F. Yvon \& Y. P. Zinchenko (dirs.), Vygotsky, une théorie du développement et de l'éducation: Recueil de textes et commentaires (pp. 299-316). MGU.

Fabbri, L. (2010). Comunità di pratiche e apprendimento riflessivo. Per una formazione situata. Carocci.

Forget, A. (2018). Penser la différenciation pédagogique. Université de Genève, [Faculté de psychologie et des sciences de l'éducation], Section des sciences de l'éducation.

Fox, E., \& Riconscente, M. (2008). Metacognition and Self-Regulation in James, Piaget, and Vygotsky. Educational Psychology Review, 20(4), 373-389. 
Revue internationale du CRIRES: innover dans la tradition de Vygotsky (2021) ISSN 2291-6717, vol 5, no 2, 36-62

Approches et méthodologies d'inspiration Vygotskiennes auprès de l'Haute École Spécialisée de la Suisse Italienne

Galand, B., Bourgeois, E., Frenay, M., \& Bentein, K. (2008). Apprentissage par problème et apprentissage coopératif: Vers une intégration fructueuse? In Y. Rouiller \& K. Lehraus (Eds.), Vers des apprentissages en coopération: Rencontres et perspectives (pp. 139-163). Peter Lang.

Gardner, H. (2008). Les intelligences multiples: La théorie qui bouleverse nos idées reçues. Retz.

Gaussel, M. (2016). Ce que la recherche nous dit sur les styles d'apprentissage (ou retour sur un mythe tenace). Édupass. https://edupass.hypotheses.org/1049

Grangeat, M. (2016). Lev S. Vygotski: L'apprentissage par le groupe. Dans M. Fournier (Ed.), Éduquer et former (pp. 134-138). Sciences Humaines.

Hébrard, P. (2013). Quelle "approche par les compétences» et quels référentiels pour la formation professionnelle aux métiers de la relation humaine? Les dossiers des sciences de l'éducation, 30, $17-34$.

Johnson, D. W., Johnson, R. T., \& Holubec, E. J. (1994). Cooperative learning in the classroom. Association for Supervision and Curriculum Development.

Lalancette, R. (2014). L'étude de cas en tant que stratégie pédagogique aux études supérieures : Recension critique. Livres en ligne du CRIRES. http://lel.crires.ulaval.ca/public/etude_de_cas_strategie.pdf

Lavoie, L., Marquis, D., \& Laurin, P. (1996). La recherche-action: Théorie et pratique: manuel d'autoformation. Presses de l'Université du Québec.

Le Boterf, G. (2018). Développer et mettre en oeuvre la compétence. Eyrolles.

Leone, L., \& Prezza, M. (2016). Costruire e valutare i progetti nel sociale. Manuale operativo per chi lavora su progetti in campo sanitario, sociale, educativo e culturale (7a edizione). Franco Angeli.

Lipari, D., \& Valentini, P. (2013). Comunità di pratica in pratica. Palinsesto.

Lotti, A. (2018). Problem-Based Learning: Apprendere per problemi a scuola: guida al PBL per l'insegnante. Franco Angeli.

Martineau, S., \& Buysse, A. A. J. (2016). Rousseau et l'éducation: Apports et tensions. Phronesis, 5(2), $14-22$.

Martini, E. R., \& Sequi, R. (1999). Il lavoro nella comunità: Manuale per la formazione e l'aggiornamento dell'operatore sociale. Carocci.

Meirieu, P. (1990). Enseigner, scénario pour un métier nouveau. ESF Editeur.

Meirieu, P. (2017). Apprendre... Oui, mais comment. ESF Editeur.

Miller, W. R., \& Rollnick, S. (2013). Motivational interviewing: Helping people change. Guilford Press.

Morin, E. (1995). La stratégie de reliance pour l'intelligence de la complexité. Revue Internationale de Systémique, 9(2), 105-112.

Morin, E. (2008). La méthode. Éd. du Seuil.

Motoi, I. (2016). La pensée critique du point de vue du travail social: Sciences \& Actions Sociales, $N^{\circ}$ 5(3), 5-32.

Mottier Lopez, L. (2016). La compétence à l'école pensée à partir de la perspective située de l'apprentissage. Éducation et Francophonie, XLIV(2), 152-171.

Perrenoud, P. (s.d.). La dimension réflexive dans l'enseignement et d'autres métiers de l'humain [Université de http://www.unige.ch/fapse/SSE/teachers/perrenoud/php_main/extraits/pratique_reflexive.html

Poikela, E., \& Poikela, S. (2005). PBL in context: Bridging work and education. Tampere University Press.

Poletti, F. (2016). La peer education in una prospettiva pedagogica. Orientamenti Pedagogici, 63(1), 187210.

Poletti, F. (2020). La Scuola Universitaria Professionale della Svizzera Italiana, nel panorama universitario svizzero. Dans A. Lotti \& P. A. Lampugnani (dirs.), Faculty Development in Italia. Valorizzazione delle competenze didattiche dei docenti universitari (pp. 41-65). Genova University Press.

Poumay, M., Tardif, J., \& Georges, F. (2017). Organiser la formation à partir des compétences: Un pari gagnant pour l'apprentissage dans le supérieur. De Boeck.

Rousseau, J.-J. (1992). Émile ou de l'éducation (éd. or. 1762). Bordas.

Sambell, K., McDowell, L., \& Montgomery, C. (2013). Assessment for learning in higher education. Routledge. 
Revue internationale du CRIRES: innover dans la tradition de Vygotsky (2021) ISSN 2291-6717, vol 5, no 2, 36-62

Approches et méthodologies d'inspiration Vygotskiennes auprès de l'Haute École Spécialisée de la Suisse Italienne

Schön, D. (1994). Le praticien réflexif. A la recherche du savoir caché dans l'agir professionnel. Les Éditions Logiques.

Schön, D. (Ed.). (1996). Le tournant réflexif: Pratiques éducatives et études de cas. Les Éditions Logiques.

Scott, C. L. (2015). Les Apprentissages de demain 3: Quel type de pédagogie pour le XXIe siècle? UNESCO Digital Library. https://unesdoc.unesco.org/ark:/48223/pf0000243126 fre

Service de soutien à la formation. (2015, décembre). Le fin mot: Différenciation pédagogique - Service de soutien à la formation - Université de Sherbrooke. https://www.usherbrooke.ca/ssf/veille/perspectives-ssf/numeros-precedents/decembre-2015/lefin-mot-differenciation-pedagogique/

Steiner, S., Stromwall, L. K., Brzuzy, S., \& Gerdes, K. (1999). Using Cooperative Learning Strategies in Social Work Education. Journal of Social Work Education, 35(2), 253-264.

Tardif, J. (2006). L'évaluation des compétences: Documenter le parcours de développement. Chenelière Education.

Tobin, T. J., \& Behling, K. (2018). Reach everyone, teach everyone: Universal design for learning in higher education. West Virginia University Press.

Tomlinson, C. A. (2014). The differentiated classroom: Responding to the needs of all learners (2nd edition). ASCD.

Venet, M., Correa Molina, E., \& Saussez, F. (2016). Pédagogie universitaire et accompagnement dans la zone proximale de développement des enseignants et enseignantes en formation initiale et continue. Nouveaux cahiers de la recherche en éducation, 19(1), 1-10.

Vygotsky, L. S. (1933). Analyse paidologique du processus pédagogique. Dans F. Yvon \& Y. P. Zinchenko (Eds.), Vygotsky, une théorie du développement et de l'éducation: Recueil de textes et commentaires (pp. 139-169). MGU.

Vygotsky, L. S. (1934a). Le problème de l'apprentissage et du développement intellectuel à l'âge scolaire. Dans F. Yvon \& Y. P. Zinchenko (dirs.), Vygotsky, une théorie du développement et de l'éducation: Recueil de textes et commentaires (pp. 221-247). MGU.

Vygotsky, L. S. (1934b). Pensée et language (3ème édition). La Dispute.

Vygotskij, L. S. (2006). Psicologia pedagogica: Manuale di psicologia applicata all'insegnamento $e$ all'educazione. Erickson.

Wenger, E. (1998). Communities of Practice: Learning, Meaning, and Identity. Cambridge University Press.

Wenger, E. (2005). La théorie des communautés de pratique. Apprentissage, sens et identité. Presses de l’Université Laval.

Yale Center for Teaching and Learning. (s.d.). Encouraging Metacognition in the Classroom Center for Teaching and Learning. https://poorvucenter.yale.edu/MetacognitioninClassrooms

Yvon, F. (2011). Penser la formation professionnelle avec Vygotsky. Dans F. Yvon \& Y. P. Zinchenko (Eds.), Vygotsky, une théorie du développement et de l'éducation: Recueil de textes et commentaires. MGU. 
Revue internationale du CRIRES: innover dans la tradition de Vygotsky (2021) ISSN 2291-6717, vol 5, no 2, 36-62 Approches et méthodologies d'inspiration Vygotskiennes auprès de l'Haute École Spécialisée de la Suisse Italienne

\section{Annexe A}

Exemple de PBL

Bachelor en physiothérapie : troisième semestre, deuxième année de formation

Module: Soins et readaptation des adultes
atteints de dysfonctionnement
neuromoteur chronique 1

Sandro, homme de 55 ans, jardinier indépendant, vit avec sa femme dans un appartement au Tessin.

Le fils de 30 ans réside avec sa femme à Genève, où il travaille.

Pendant l'entretien initial, Sandro vous informe qu'il a récemment appris qu'il souffrait de la maladie de Parkinson (grade II avancé, échelle de Hoehn \& Yahr).

Il raconte qu'il s'est adressé au médecin parce qu'il s'est rendu compte qu'il avait des difficultés à effectuer des activités telles que : utiliser des ciseaux de jardin, biner avec un petit outil qui demande précision, se raser avec précision, fermer/desserrer les boutons ...

Sandro fréquent un gymnase où il suit des cours de gymnastique. Ces derniers temps, il éprouve des difficultés à suivre le groupe surtout dans les exercices de coordination et de vitesse.

Lorsqu'il marche, il dit se sentir "raide, fatigué", et sur un sol irrégulier, il a remarqué qu'il avait tendance à perdre facilement l'équilibre ; pour cette raison, il doit marcher lentement et faire attention à ce qu'il fait.

En outre, il a dû ajouter un oreiller sous la nuque afin de pouvoir dormir détendu dans son lit.

Dernièrement, il rapporte se sentir plutôt triste et apathique sans raisons apparentes (le travail va bien et les relations affectives aussi). En outre, au travail, il se sent plus fatigué au niveau de l'attention.

Sa femme et ses amis ont remarqué son apathie : il ne prend aucune initiative et il semble avoir peu d'émotions (que peu le sollicite émotionnellement), ses expressions ne sont plus ce qu'elles étaient.

Actuellement, cela n'a pas d'impact important au niveau de la participation (ICF), malgré son malaise subjectif.

Lors de l'évaluation, vous notez que les aspects posturaux pendant la marche sont caractérisés par la flexion des genoux, des hanches et du tronc, et que la tête est placée devant et contrainte par les mouvements du tronc.

Les paramètres du chemin sont relativement physiologiques, mais vous observez que la longueur de la foulée est courte.

Lors des tests d'équilibre, les réponses aux perturbations externes sont lentes et inefficaces.

Dans les changements de direction les stratégies distales et de la hanche semblent être incoordonnées.

En décubitus dorsal, vous observez que l'attitude de flexion du tronc et des membres inférieurs demeure. Lors de la mobilisation passive, bien que l'amplitude articulaire soit maintenue, on perçoit une altération du tonus dans le sens de l'hypertone.

Vous remarquez des mouvements involontaires à la main droite quand il est au repos.

Expliquez le tableau clinique de Sandro.

Définissez des objectifs de traitement pertinents et des propositions thérapeutiques.

\section{Références bibliographiques pour l'étude autonome}

Cambier J., M. Masson, H. Dehen Neurologia, Milano, Masson, 2005

- Capitolo 2 Semiologia della motilità

- Capitolo 15 Sindromi parkinsoniane

Purves D., G.J. Augustine, D. Fitzpatrick, W.C. Hall, A.S. LaMantia, J.O. McNamara, L.E.White, 2009, Neuroscienze, Bologna, Zanichelli

\section{Documents PDF chargés sur la platforme éléctronique Moodle}

Bonaiuti Donatella Scale di misura per il morbo di Parkinson in Le scale di misura in 
Revue internationale du CRIRES: innover dans la tradition de Vygotsky (2021) ISSN 2291-6717, vol 5, no 2, 36-62 Approches et méthodologies d'inspiration Vygotskiennes auprès de l'Haute École Spécialisée de la Suisse Italienne

riabilitazione, Roma,Società Editrice Universo, 2005

\section{Bibliographie pour approfondir le thème}

Schoenhuber R et all. Qualità di vita nel morbo di Parkinson, Neurol Sci (2004) 25:S 103-S105

Parkinson's disease Society, 2007 The professional guide of Parkinson's disease con particolare riferimentoal capitoloThe physiotherapist's guide pagine 103-129

Linee guida sulla gestione del paziente disfagico adulto in foniatria e logopedia, Federazione LogopedistiItaliani, 29 Gennaio 2007

Esercizi per la rieducazione del linguaggio nel morbo di Parkinson (pubblicazione a cura dell'unioneParkinsoniani)

Dalla parte del paziente: brani tratti dal testo di Michela Cancelliere Miss Parkinson, S. Paolo, Alba, 2007 


\section{Annexe B}

\section{Bachelor en travail social : cinquième semestre, troisième année de formation}

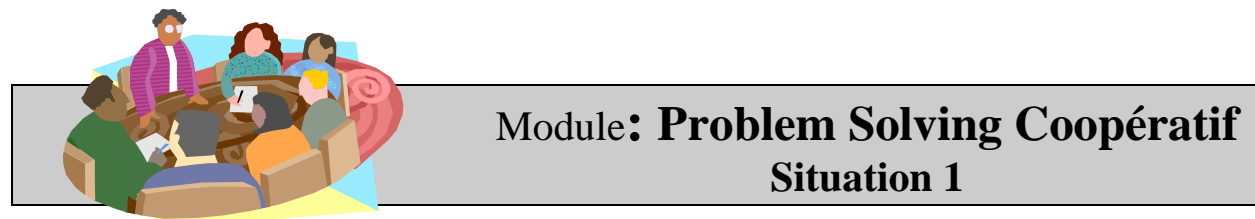

On est début juin.

Vous représentez l'équipe du "Foyer Libre" qui accueille actuellement 8 enfants en âge de scolarité obligatoire. Le groupe d'enfants est composé comme suit:

- un garçon de 13 ans (collège : deuxième année);

- une fille de 11 ans (collège : première année);

- une fille de 15 ans (collège : quatrième année);

- deux frères jumeaux de 9 ans (école primaire : quatrième année);

- une petite-fille de 7 ans (école primaire : deuxième année);

- deux garçons de 8 ans (école primaire : deuxième et troisième années).

Ludovico est un enfant de 6 ans et demi et entrera dans le foyer début août, un mois avant le début de l'année scolaire.

Les nouvelles que vous avez reçues sur la situation de Ludovico par le service d'envoi sont les suivantes:

La mère de Ludovico, divorcée depuis des années du père de l'enfant, a eu dans le passé de sérieux problèmes d'abus de drogue.

Le père ne s'est jamais intéressé à Ludovico et il ne veut pas le faire maintenant.

Depuis quelques années la mère vit avec un nouveau compagnon, mais au cours de la dernière année la relation entre les deux est devenue conflictuelle.

La femme présente également d'importants problèmes de santé qui la fragilisent sur le plan de l'autonomie.

Le choix de l'insertion de Ludovico dans le foyer est la conséquence de cette situation.

La structure d'accueil est située dans une petite maison d'un village à une dizaine de kilomètres du domicile de l'enfant. Le foyer reste ouvert toute l'année. Il y a une équipe de 6 éducateurs qui couvrent tous les postes. Compte tenu des graves problèmes de santé de la mère et de l'insuffisance éducative de l'environnement familial, l'insertion implique un placement à long terme.

L’équipe a pour mission de préparer l'insertion de Ludovico dans le foyer.

\section{Tâches à accomplir par les groupes}

Pendant les réunions qui se tiendront pendant les cours du (date1) au (date2), nous vous demandons de:

1. envisager l'organisation de l'insertion de Ludovico, en tenant compte de sa situation, des exigences de la structure, d'autres enfants et de sa famille ;

2. envisager le projet éducatif qui pourrait être mis en œuvre à l'intérieur de la structure avec Ludovico ;

3. organiser une présentation de 10 minutes à effectuer à la fin de la réunion de la date 2 : choix effectués, motivations, parcours de résolution de problèmes expérimenté. 


\section{Annexe C}

\section{Bachelor en travail social : cinquième semestre, troisième année de formation}

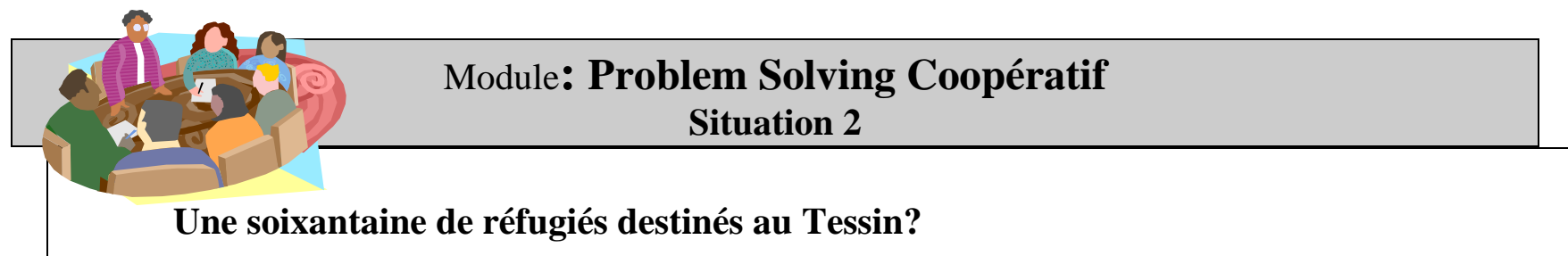

Un pourcentage des 1500 réfugiés destinés à la Suisse finira dans le sud des Alpes.

Hier la Confédération a annoncé d'avoir adhéré au programme de relocalisation de 40'000 personnes nécessiteuses de protection, lancé de l'Union européenne (EU) en juillet. Le Conseil fédéral a en effet décidé d'accueillir jusqu'à 1500 personnes déjà enregistrées en Italie et en Grèce.

Les réfugiés seront répartis entre les différents Cantons et une soixantaine, selon la RSI [Radiotélévision de la Suisse italienne], pourraient bientôt arriver au Tessin. Il s'agirait de 3,9\% des 1500 réfugiés, pour lesquels une cellule opérationnelle composée de représentants du Département des Institutions, du Département de la Santé et des affaires sociales et de l'Office fédéral des migrations se serait déjà mise au travail. "Il s'agit d'un groupe de travail qui s'occupera surtout d'organiser l'hébergement de ces personnes dans des délais rapides », a précisé le ministre Paolo Beltraminelli.

ticinonews-journal online | 19 set $201514: 21$

Les personnes originaires de Syrie seront principalement constituées de ménages.

Après leur arrivée en Suisse, les réfugiés passent une dizaine de jours dans un centre d'enregistrement et de procédure de la Confédération, après quoi ils sont transférés dans le Canton d'affectation, où l'asile leur est accordé en application de l'article 56 de la Loi sur l'asile. En Suisse, les réfugiés ne sont donc pas soumis à une véritable procédure d'asile. L'intégration dans le Canton d'accueil se fait dans le cadre des mesures d'intégration ordinaire. Le regroupement familial n'est possible que pour les enfants mineurs et les conjoints qui ont été séparés au moment de la fuite. En règle générale, on essaie d'accueillir toute la famille dans le cadre de la relocalisation.

En particulier dans notre Canton, les personnes accueillies, après être passées par un centre d'enregistrement (Chiasso ou autre Canton) seront introduites dans les centres de la Croix-Rouge et, en fonction de la disponibilité de logements, seront ensuite prises en charge par SOS Ticino qui les insérera dans des appartements sur le territoire.

Il est très probable que le groupe de réfugiés sera accueilli par les communes de X, Y, Z. Ceux-ci ont donné leur disponibilité pour les loger dans des structures publiques et privées.

Le Canton a donné mandat à une association présente sur le territoire d'élaborer un projet visant à promouvoir l'accueil et à activer un processus d'intégration avec ces personnes sur notre territoire. La nouvelle "Association Prosiria", fondée par un groupe de travailleurs sociaux qui opère déjà dans le Canton dans des domaines divers, a décidé de promouvoir une série d'initiatives visant à favoriser l'échange et l'intégration entre la population des municipalités concernées et les familles syriennes.

Mise en situation : vous êtes les membres de l'Association Prosiria

Questions à traiter et à discuter :

1. Qui sont ces gens ? Quels sont leurs besoins ? Que peut-on faire concrètement ? Et comment ?

2. Entre les différents organismes, services, agences, qui est impliqué? Quel travail de réseau est possible ?

3. Quelle est la représentation de la population de ces personnes ?

4. Les représentations de tous les acteurs concernés ont-elles une influence réciproque ? 
Revue internationale du CRIRES: innover dans la tradition de Vygotsky (2021) ISSN 2291-6717, vol 5, no 2, 36-62 Approches et méthodologies d'inspiration Vygotskiennes auprès de l'Haute École Spécialisée de la Suisse Italienne

\section{Tâches à accomplir par les groupes}

Pendant les réunions qui se tiendront pendant les cours et celles que vous organisez de façon autonome, nous vous demandons de:

- Élaborer une proposition opérationnelle qui réponde aux termes du problème tel que décrit ci-dessus en tenant compte de tous les aspects principaux.

- Organiser, pour le dernier jour du cours, la présentation de votre projet en plénière (temps disponible 20 minutes). La présentation devra prévoir le contenu de votre proposition de projet et le parcours de résolution de problèmes expérimenté (aspects de contenu et dynamiques relationnelles). 